\title{
Identification of proteins associated with paclitaxel resistance of epithelial ovarian cancer using iTRAQ-based proteomics
}

\author{
YUANJING WANG and HONGXIA LI
}

\begin{abstract}
Department of Obstetrics and Gynecology, Beijing Shijitan Hospital, Capital Medical University, Beijing 100038, P.R. China
\end{abstract}
Received February 20, 2017; Accepted October 26, 2017

DOI: $10.3892 / \mathrm{ol} .2018 .8600$

\begin{abstract}
Chemotherapy is an important adjuvant therapy for epithelial ovarian cancer (EOC). The main cause of chemotherapy failure in EOC is paclitaxel resistance. The present study aimed to identify novel biomarkers to predict chemosensitivity to paclitaxel and improve our understanding of the molecular mechanisms underlying paclitaxel resistance in EOC. In the present study, the heterogeneity of EOC was evaluated by adenosine triphosphate-tumor chemosensitivity assay (ATP-TCA) in vitro. Fresh samples were collected from 54 EOC cases during cytoreductive surgery. Tumor cells were isolated, cultured, and tested for sensitivity to paclitaxel. Proteins that were differentially expressed between paclitaxel-resistant tissues and paclitaxel-sensitive tissues were identified via isobaric tags for relative and absolute quantitation (iTRAQ)-based proteomic analysis. Two upregulated proteins, plexin domain containing 2 (Plxdc2) and cytokeratin 7 (CK7), were selected to verify the iTRAQ method using western blot analysis in EOC tissues with different chemosensitivities (sensitive, weakly sensitive and resistant). There was notable heterogeneity of chemosensitivity in the EOC specimens. Highly to mildly-differentiated or early-stage (I/II) EOC specimens had decreased sensitivity to paclitaxel compared with specimens with low differentiation $(\mathrm{P}<0.05)$ or an advanced stage (III; $\mathrm{P}<0.05)$, respectively. A total of 496 significantly differentially expressed proteins, including 263 that were downregulated $(\mathrm{P}<0.05)$ and 233 that were upregulated $(\mathrm{P}<0.05)$ in paclitaxel-resistant tissues compared with paclitaxel-sensitive tissues, were identified using iTRAQ in combination with LC-MS/MS. The expression levels of two proteins associated with paclitaxel resistance, Plxdc2 and CK7, were further validated by western blotting, which revealed that they were upregulated in the paclitaxel-resistant tissues. The
\end{abstract}

Correspondence to: Dr Hongxia Li, Department of Obstetrics and Gynecology, Beijing Shijitan Hospital, Capital Medical University, 10 Tieyi Road, Beijing 100038, P.R. China

E-mail: lihongxiavs@163.com

Key words: ovarian cancer, paclitaxel resistance, plexin domain containing 2, cytokeratin 7, isobaric tags for relative and absolute quantitation present study determined candidate proteins associated with paclitaxel resistance in EOC. Plxdc2 and CK7 may be potential makers for distinguishing patients with paclitaxel-resistant EOC from those with paclitaxel-sensitive EOC.

\section{Introduction}

Ovarian cancer is the most lethal gynecological malignancy in the world (1). Due to the limited number of specific symptoms, women usually only seek medical help once the disease is at an advanced stage, with distant metastases (2). Overall, $90 \%$ of ovarian cancer cases are epithelial ovarian cancer (EOC) (3). Standard therapy for advanced EOC involves a combination of cytoreductive surgery and platinum-based chemotherapy, with the combination of paclitaxel and platinum being the standard adjuvant chemotherapy regimen for EOC (4). Paclitaxel is an important agent for EOC treatment, and is an effective first-line therapy for advanced ovarian cancer. However, recurrence still affects the majority of patients a short period following chemotherapeutic intervention (5). The main cause for the failure of chemotherapy is chemoresistance of the tumor tissues, which adversely affects the prognosis of patients with ovarian cancer. Furthermore, patients with ovarian carcinoma may have variable responses to the standard chemotherapeutic regimen, even when they have the same histologic type. Heterogeneity of the tumor tissue, one of the primary features of malignancies, is thought to be the main factor causing this difference (6). With the incidence of paclitaxel resistance increasing, it is necessary to identify novel, specific biomarkers that predict chemosensitivity to paclitaxel to improve outcomes for patients with ovarian cancer $(7,8)$.

The chemosensitivity test is an in vitro, predictive assay for used to assess cancer cell sensitivity to a range of chemotherapeutic agents. Adenosine triphosphate-tumor chemosensitivity assays (ATP-TCA) are sensitive assays that have been widely used to determine the drug sensitivity of solid tumors in the past few years (9). ATP-TCA measures the intracellular ATP levels of drug-exposed cells and untreated controls to assess tumor cell viability. This method has notable advantages for guiding the design of chemotherapy protocols and individualized treatments, and assessing novel chemotherapeutic drugs. Since its introduction, a number of studies have reported that ATP-TCA have a high sensitivity and a positive predictive value, and accurately predict the response to chemotherapy in ovarian cancer $(10,11)$. 
In the present study, ATP-TCA was used to assess the chemosensitivity of EOC to paclitaxel. Parameters determined by analyzing the correlation between the inhibition rate and paclitaxel doses were measured as follows: Inhibitory concentration (IC) $)_{90}$ and $\mathrm{IC}_{50}$, (90 or $50 \%$ growth inhibition in vitro, respectively), and sensitivity index (SI), which was calculated by summation of the percentage of tumor growth inhibition (TGI) at each concentration detected (12). SI >250 was suggested to be the optimal standard for predicting chemoresistance. Therefore, 250 were selected as the cut-off point for SI in the present study (13).

Paclitaxel is known to induce cytotoxicity by triggering apoptosis via regulation of the expression of apoptosis-associated proteins in the caspase-independent and caspase-dependent pathways, or by preventing tubulin depolymerization during the metaphase to anaphase transition of mitosis (14). However, paclitaxel resistance limits its use in the long-term management of EOC, and the molecular mechanisms underlying this resistance remain to be fully elucidated. Therefore, the identification of specific markers for ovarian cancer with paclitaxel resistance is a long-term goal of the medical community. The present study aimed to identify proteins associated with paclitaxel resistance in ovarian cancer, in order to investigate the molecular mechanisms underlying paclitaxel resistance and discover potential novel drug targets for paclitaxel-resistant ovarian cancer (15).

In the present study, two approaches for quantitative proteomic analysis were selected for identifying the differentially expressed proteins between paclitaxel-resistant and paclitaxel-sensitive groups of ovarian cancer tissues: iTRAQ analysis and two-dimensional electrophoresis coupled to liquid chromatography tandem mass spectrometry (LC-MS/MS). iTRAQ is a gel free mass spectrometry technique, applying isobaric amine specific tags to compare peptide intensities between samples, then inferring quantitative values for the corresponding proteins. LC-MS/MS is based on the differential two-dimensional gel electrophoresis pattern between protein samples and provides additional biological information, including molecular weight alterations or isoelectric point drift, based on which protein functions are implicated (16). The present study aimed to identify biomarkers, which were associated with paclitaxel-resistant ovarian cancer, providing information to aid our understanding of the underlying molecular mechanisms and to predict treatment responses to therapeutic agents. The ovarian cancer-specific proteins identified were further confirmed by western blot analysis (17).

\section{Materials and methods}

Ethics statement. The study protocol received approval from the Ethics Committee of the Beijing Shijitan Hospital, Capital Medical University (Beijing, China). Written, signed informed consent was obtained from all patients and their family members prior to surgery. All procedures were carried out in agreement with the Code of Ethics of the World Medical Association (Declaration of Helsinki, 1964; as revised in 2004).

Tumor samples. A total of 54 fresh specimens were obtained from patients with EOC who underwent staging surgery at the
Beijing Shijitan Hospital, Beijing University People's Hospital (Beijing, China), People's Liberation Army General Hospital (Beijing, China) and Beijing Obstetrics and Gynecology Hospital (Beijing, China), between March 2013 and December 2014. Routine histopathology was conducted on formalin-fixed and paraffin-embedded samples, which were obtained from the same tissues, by at least two experienced gynecological pathologists (the Beijing Shijitan Hospital) in order to determine the malignancy and the stage of the tumor samples. Each fresh collected sample was divided into two fractions: One was prepared for ATP-TCA, and the other was stored at $-80^{\circ} \mathrm{C}$ for subsequent tests. The ATP-TCA was conducted as a routine procedure immediately following surgery using residual primary tumor samples which were not required for histopathology. The sensitivity of viable ovarian cancer cells harvested from malignant tissues to paclitaxel (Corden Pharma Latina S.P.A., Sermoneta Italy) was then detected as follows.

In vitro ATP-TCA. An ATP-TCA kit, containing serum-free complete assay medium, digestive enzyme and luciferin-luciferase reagent (Huzhou Haichuang Biotech Co., Ltd., Huzhou, China) was used for the assessment of chemosensitivity. The ATP-TCA was performed as previously described $(12,18)$. Briefly, samples (1-2 $\left.\mathrm{cm}^{3}\right)$ were harvested from solid tumors during surgical resection and cut into smaller fragments $\left(1 \mathrm{~mm}^{3}\right)$. The fragments were then incubated with $5-10 \mathrm{ml}$ sterile digestive enzyme reagent for $2-3 \mathrm{~h}$ at $37^{\circ} \mathrm{C}$ in a $5 \% \mathrm{CO}_{2}$ incubator, and dissociated to form a single cell suspension. Once the concentration of the cell suspension was adjusted to $2-4 \times 10^{5} / \mathrm{ml}, 100 \mu \mathrm{l}$ cell suspension was seeded into a 96-well polypropylene microplate. Cells were incubated with $5 \% \mathrm{CO}_{2}$ at $37^{\circ} \mathrm{C}$ for 5 days, and treated with five different doses $(12.5$, $25,50,100$ and $200 \%$ ) of the test drug concentration (TDC) derived from the plasma peak concentrations, which were in turn determined by pharmacokinetic and clinical information (19). The standard $100 \%$ TDC value of paclitaxel was $13.8 \mathrm{~g} / \mathrm{ml}$. The assay was performed in duplicate wells, with positive and negative controls. For each dose, two controls were included in each plate: A drug free control comprised of media only (M0) and a maximum inhibitor (MI) control which kills all cells present. At the end of the 5 day-incubation, the cells were lysed with $50 \mu \mathrm{l}$ ATP extraction reagent, and $50 \mu \mathrm{l}$ luciferin-luciferase reagent was added to each well. A luminometer (Orion II; Berthold Technologies GmbH \& Co. $\mathrm{KG}$, Bad Wildbad, Germany) was used to assess the level of ATP present, and an inhibition curve was plotted.

iTRAQ combined with $L C-M S / M S$. According to the results of the ATP-TCA, tumor specimens were divided into three main types: Sensitive, weakly sensitive and resistant. In order to screen the altered proteins associated with paclitaxel resistance more effectively, sensitive specimens ( $\mathrm{S}$ group, $\mathrm{n}=8$ ) and resistant specimens ( $\mathrm{R}$ group, $\mathrm{n}=8$ ) were selected for iTRAQ analysis. Frozen tissues were homogenized and sonicated (20 kHz) using $0.5 \%$ sodium dodecyl sulfate (SDS) with a cell disperser, followed by centrifugation at $20,000 \mathrm{x} g$ for $30 \mathrm{~min}$ at $4^{\circ} \mathrm{C}$ to eliminate the cell debris. Following this, the supernatant was collected, and the Bradford assay was used to determine protein concentration. Next, $100 \mu \mathrm{g}$ protein per condition were treated with dithiothreitol $(10 \mathrm{mM})$ and iodoacetamide 
(55 $\mathrm{mM}$ ) for reduction and alkylation. Following this, the proteins were digested with trypsin (Promega Corporation, Madison, WI, USA), and the resultant peptides mixture was further labeled using chemicals from the iTRAQ reagent kit (Applied Biosystems; Thermo Fisher Scientific, Inc., Waltham, MA, USA) according to the manufacturer's protocols. The samples were marked with iTRAQ tags as follows: iTRAQ115 for the S group and iTRAQ116 for the R group.

Next, the iTRAQ-labeled peptides were pooled and fractionated by strong cation exchange (SCX) chromatography on a SCX column $(5 \mu \mathrm{m}, 100 \mathrm{~A}$; Phenomenex, Torrance, CA, USA) with a linear gradient from $0 \% \mathrm{~B}$ to $100 \% \mathrm{~B}$ in $90 \mathrm{~min}$ at a flow rate of $1 \mathrm{ml} / \mathrm{min}$ (solution $\mathrm{A}: 10 \mathrm{mM}$ $\mathrm{KH}_{2} \mathrm{PO}_{4}$, pH 3.0, 25\% acetonitrile; solution $\mathrm{B}: 2 \mathrm{M} \mathrm{KCl}$, $10 \mathrm{mM} \mathrm{KH}{ }_{2} \mathrm{PO}_{4}, \mathrm{pH} \mathrm{3.0,25 \%}$ acetonitrile). According to the chromatography results, the collected fractions were recombined into 16 fractions and then freeze-dried $\left(-10^{\circ} \mathrm{C}\right)$. Following this, each freeze-dried fraction from the SCX column was re-dissolved in $100 \mu 10.1 \%$ formic acid aqueous solution, and then desalted using a strata-X C18 column (Phenomenex). The sample was then extracted and analyzed using nano-LC-MS/MS with a quadrupole-Orbitrap mass spectrometer (Q-Exactive; Thermo Fisher Scientific, Inc.) as previously described (20).

Western blot analysis. Based on the proteomic results, two proteins of interest, plexin domain containing 2 (Plxdc2) and cytokeratin 7 (CK7), were expressed at higher levels in paclitaxel-resistant tissues than paclitaxel-sensitive tissues. Western blot analysis was used to examine the expression of CK7 and Plxdc2 in EOC tissues with different chemosensitivities (sensitive, weakly sensitive and resistant). The protein selections were based on a high fold change (FC) and high significance (Plxdc2, $\mathrm{P}<0.05 ; \mathrm{FC}=1.539 ; \mathrm{CK} 7, \mathrm{P}<0.05 ; \mathrm{FC}=1.724)$. The extracted proteins $(20 \mu \mathrm{g})$ were separated by $12 \%$ SDS-PAGE and transferred onto nitrocellulose membranes. Following blocking with 5\% non-fat milk in Tris-buffered saline with $0.1 \%$ Tween-20 at room temperature for $1 \mathrm{~h}$, the membranes were probed with the following primary antibodies: Rabbit anti-human polyclonal Plxdc2 (dilution, 1:5,000; cat. no. NBP1-76858; Novus Biologicals, LLC, Littleton, CO, USA) and rabbit anti-human polyclonal CK7 (dilution, 1:10,000; cat. no. ab154334; Abcam, Cambridge, MA, USA) at $4^{\circ} \mathrm{C}$ overnight. Following washing with Tris-buffered saline with Tween-20 three times, the membranes were incubated with a horseradish peroxidase-conjugated goat anti-rabbit immunoglobulin $\mathrm{G}$ antibody (dilution, 1:5,000; cat. no. ab97051; Abcam, Cambridge, MA, USA) at room temperature for 1-2 h. Membranes were washed as aforementioned and analyzed using a two-color infrared imaging system (Odyssey; Li-COR Biosciences, Lincoln, NE, USA). The gray level of each band was calculated using image processing ImageJ software (version 1.48; National Institutes of Health, Bethesda, MA, USA). Densitometric analysis of the bands was conducted three times and normalized to GAPDH (dilution, 1:5,000; cat. no. ab9485; Abcam, Cambridge, MA, USA).

\section{Data analysis}

ATP-TCA. ATP-TCA data were exported to a Microsoft Excel 2010 spreadsheet (Microsoft Corporation, Redmond, WA,
Table I. Characteristics of tumor samples $(n=54)$.

\begin{tabular}{lrr}
\hline Characteristics & $\mathrm{N}$ & $(\%)$ \\
\hline Histology & & \\
Serous & 41 & 75.9 \\
Mucinous & 2 & 3.7 \\
Clear cell & 5 & 9.3 \\
Endometrioid & 4 & 7.4 \\
Transitional cell & 2 & 3.7 \\
FIGO stage & & \\
I & 8 & 14.8 \\
II & 7 & 13.0 \\
III & 39 & 72.2 \\
Grade of differentiation & & \\
High & 5 & 9.3 \\
Mild & 7 & 73.0 \\
Low & 42 & 88.9 \\
Primary & 48 & 11.1 \\
Recurrent & 6 & \\
\end{tabular}

FIGO, International Federation of Gynecology and Obstetrics.

USA), and the parameters SI (SI=500 - sum of $\%$ TGI at 200, 100, 50, 25 and $12.5 \%$ TDC), $\mathrm{IC}_{90}$ and $\mathrm{IC}_{50}$ were compared. The three types of in vitro sensitivity were defined below: Sensitive (S), $\mathrm{IC}_{50}<25 \%$ TDC and $\mathrm{IC}_{90} \leq 100 \%$ TDC; weakly sensitive (WS), $\mathrm{IC}_{50}<25 \% \mathrm{TDC}$ and $\mathrm{IC}_{90} \leq 100 \% \mathrm{TDC}$ or SI $\leq 250$; and resistant (R), SI $>250$. Quality control for each assay was conducted as follows: Two measurements of every drug-treated sample were used for controlling the variability of individual ATP values. Samples with coefficient of variation $(\mathrm{CV})>0.15$ were rejected and retested. For the present study, the mean CV was 0.048 (range, 0.023-0.114).

iTRAQ assay. LC-MS/MS analysis of iTRAQ-labeled peptides was performed using Mascot (version 2.3.0) and Proteome Discoverer Version 1.3 software (Thermo Fisher Scientific, Inc.) and identification of the proteins was conducted by utilizing the raw MS data (21). For quantitative iTRAQ analysis, the peptide was automatically selected by Protein Discoverer with the Pro Group ${ }^{\mathrm{TM}}$ algorithm, and the error factor, P-value and the reporter peak area were calculated. If the ITRAQ ratio (sensitive tissues/resistant tissues) was $<0.83$ or $>1.2(\mathrm{P}<0.05)$, the protein was considered to be differentially expressed (22). Next, Gene Ontology enrichment analysis was conducted to analyze functions of the differentially expressed proteins using Bioconductor 3.0 software (https://www.bioconductor. org), and biological process, molecular function and cellular component were included. For significant enrichment of the protein sets, a false discovery rate of $<0.05$ was considered as a threshold (23-25).

Statistical analysis. All results are expressed as the mean \pm standard deviation. Statistical analysis between groups was performed using SPSS 18.0 software (SPSS, Inc., Chicago, IL, USA), and comparisons were made using an 
Table II. Associations between the adenosine triphosphate-tumor chemosensitivity assay results for paclitaxel resistance and the stage or grade of differentiation of tumor samples.

\begin{tabular}{lccccrrrr}
\hline & \multicolumn{3}{c}{ FIGO stage } & & \multicolumn{3}{c}{ Differentiation } \\
\cline { 2 - 4 } \cline { 6 - 8 } Sensitivity to paclitaxel & I/II & III & P-value & & High-mild & Low & P-value \\
\hline S+WS & 8 & 34 & 0.021 & & 5 & 37 & 0.003 \\
R & 7 & 5 & & & 7 & 5 & \\
\hline
\end{tabular}

FIGO, International Federation of Gynecology and Obstetrics; S, sensitive; WS, weak sensitive; R, resistant.
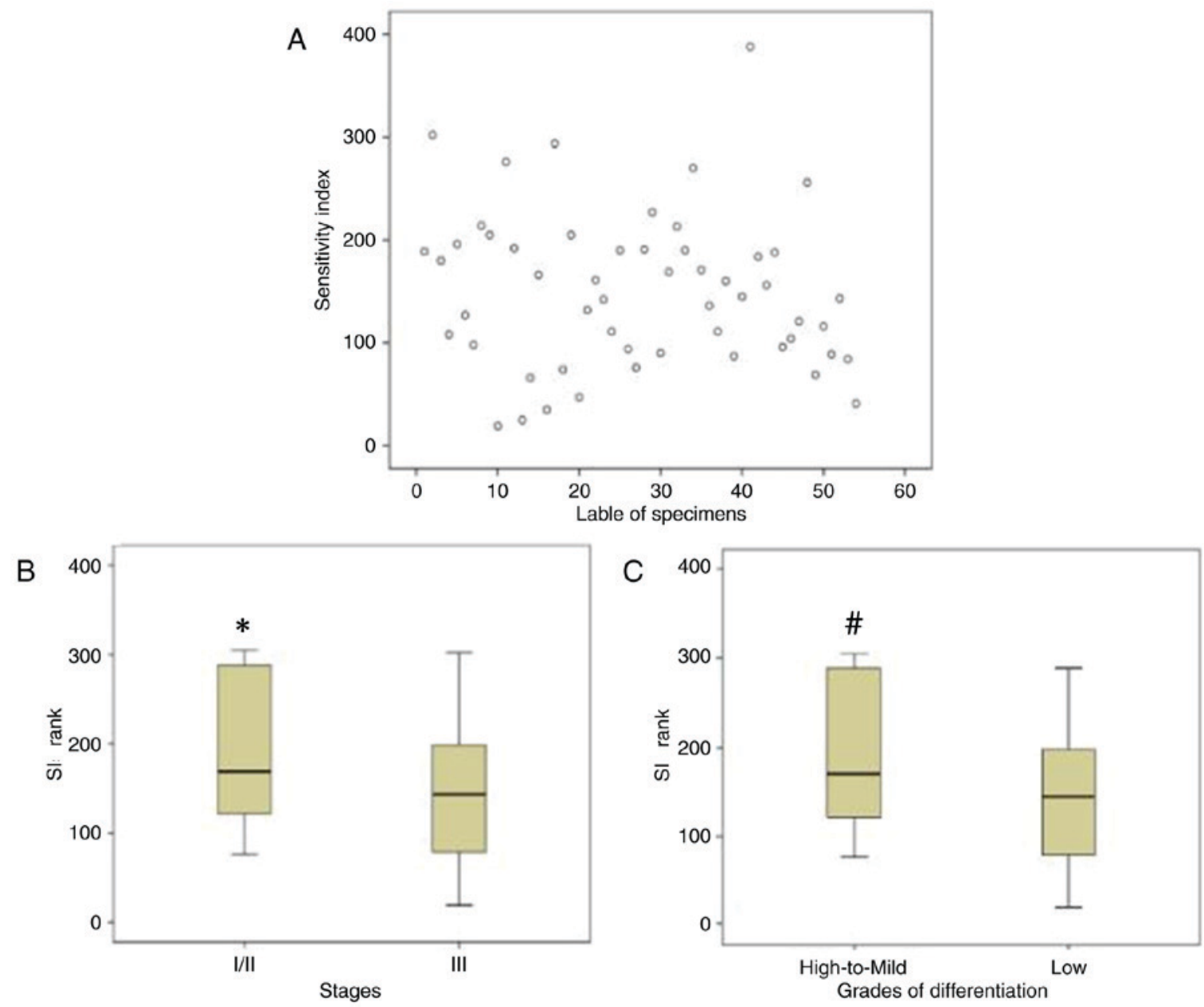

Figure 1. Results of in vitro adenosine triphosphate-tumor chemosensitivity assays. (A) Scatter plots demonstrating heterogeneity of paclitaxel in various tumor samples. Box-and-whisker plots of the SI rank of paclitaxel in specimens with different (B) stages or (C) grades of differentiation. The bottom and top edges of the boxes represent the 25 th and 75 th percentiles, respectively, whereas the horizontal line corresponds to the median value. The vertical lines show the range of values. ${ }^{*} \mathrm{P}<0.05$ vs. advanced-stage (III) specimens, ${ }^{*} \mathrm{P}<0.05$ vs. low differentiation. SI, sensitivity index.

unpaired Student's t-test, $\chi^{2}$ test and one-way analysis of variance (ANOVA). Fishers least significant difference test was performed on ANOVA data in order to determine statistical significance. $\mathrm{P}<0.05$ was considered to indicate a statistically significant difference.

\section{Results}

In vitro ATP-TCA. The patients were aged between $20-76$ years, with a median age of 51 years. The tumor characteristics of the samples are listed in Table I. Notable heterogeneity in chemosensitivity was observed among the tumor samples examined (Fig. 1A). There was a significant association between clinical indicators of the tumor samples and the ATP-TCA results. The associations between the stage or differentiation grade of the tumor samples and the ATP-TCA results were assessed using $\chi^{2}$ tests. It was demonstrated that specimens with high to mild differentiation or an early stage (I/II) had lower chemosensitivity to paclitaxel when compared with low-differentiated or advanced stage (III) specimens, respectively (Table II). 
Table III. Differentially expressed proteins in S tissues compared with R tissues.

\begin{tabular}{|c|c|c|}
\hline Serial no. & Protein & Fold-change for $\mathrm{S} / \mathrm{R}$ \\
\hline \multicolumn{3}{|c|}{ Upregulated in $\mathrm{R}$ tissues } \\
\hline P02765 & $\alpha$-2-HS-glycoprotein & 0.346 \\
\hline Q9BW30 & Tubulin polymerization-promoting protein family member 3 & 0.359 \\
\hline Q92954 & Proteoglycan 4 & 0.463 \\
\hline P00734 & Prothrombin & 0.467 \\
\hline P07602 & Proactivator polypeptide & 0.485 \\
\hline P35080 & Profilin-2 & 0.517 \\
\hline Q9UNP9 & Peptidyl-prolyl cis-trans isomerase $\mathrm{E}$ & 0.524 \\
\hline Q14508 & WAP four-disulfide core domain protein 2 & 0.525 \\
\hline Q6ZU11 & Uncharacterized protein C9orf142 & 0.539 \\
\hline Q9H6Y7 & E3 ubiquitin-protein ligase RNF167 & 0.547 \\
\hline P09758 & Tumor-associated calcium signal transducer 2 & 0.552 \\
\hline P84157 & Matrix-remodeling-associated protein 7 & 0.565 \\
\hline Q9H4G0 & Band 4.1-like protein 1 & 0.567 \\
\hline P08729 & Cytokeratin 7, type II cytoskeletal 7 & 0.580 \\
\hline P42330 & Aldo-keto reductase family 1 member C3 & 0.587 \\
\hline O75882 & Attractin & 0.592 \\
\hline Q969E4 & Transcription elongation factor A protein-like 3 & 0.595 \\
\hline Q9Y240 & C-type lectin domain family 11 , member A & 0.604 \\
\hline P05783 & Cytokeratin 7, type I cytoskeletal 18 & 0.623 \\
\hline P81605 & Dermcidin & 0.644 \\
\hline P09455 & Retinol-binding protein 1 & 0.649 \\
\hline Q6UX71 & Plexin domain-containing protein 2 & 0.650 \\
\hline O43175 & D-3-phosphoglycerate dehydrogenase & 0.651 \\
\hline P55809 & Succinyl-CoA:3-ketoacid-coenzyme A transferase 1, mitochondrial & 0.653 \\
\hline Q7L2H7 & Eukaryotic translation initiation factor 3 subunit $\mathrm{M}$ & 0.688 \\
\hline Q12805 & EGF-containing fibulin-like extracellular matrix protein 1 & 0.689 \\
\hline Q8TEQ8 & GPI ethanolamine phosphate transferase 3 & 0.691 \\
\hline Q9C0H2 & Protein tweety homolog 3 & 0.695 \\
\hline P00751 & Complement factor B & 0.698 \\
\hline Q14676 & Mediator of DNA damage checkpoint protein 1 & 0.701 \\
\hline Q9BUH6 & Uncharacterized protein C9orf142 & 0.702 \\
\hline Q9BX66 & Sorbin and SH3 domain-containing protein 1 & 0.702 \\
\hline P02786 & Transferrin receptor protein 1 & 0.706 \\
\hline P01861 & $\operatorname{Ig} \gamma-4$ chain $\mathrm{C}$ region & 0.706 \\
\hline O15305 & Phosphomannomutase 2 & 0.707 \\
\hline O43752 & Syntaxin-6 & 0.731 \\
\hline Q86SX6 & Glutaredoxin-related protein 5 & 0.732 \\
\hline Q8NFV4 & Abhydrolase domain-containing protein 11 & 0.736 \\
\hline Q14696 & LDLR chaperone MESD & 0.736 \\
\hline P17931 & Galectin-3 & 0.739 \\
\hline Q8WWF6 & DnaJ homolog subfamily B member 3 & 0.741 \\
\hline \multicolumn{3}{|c|}{ Downregulated in $\mathrm{R}$ tissues } \\
\hline Q15063 & Periostin & 2.041 \\
\hline P49913 & Cathelicidin antimicrobial peptide & 2.064 \\
\hline P41218 & Myeloid cell nuclear differentiation antigen & 2.111 \\
\hline P01814 & Ig heavy chain V-II region OU & 2.145 \\
\hline Q9HCF4 & Protein ALO17 & 2.231 \\
\hline P59665 & Neutrophil defensin 1 & 2.232 \\
\hline P05164 & Myeloperoxidase & 2.246 \\
\hline P20962 & Parathymosin & 2.283 \\
\hline P61626 & Lysozyme C & 2.284 \\
\hline
\end{tabular}


Table III. Continued.

Serial no.

Protein

Fold-change for S/R

\begin{tabular}{lll}
\hline A8MW06 & Thymosin $\beta$-4-like protein 3 & 2.329 \\
Q9NP78 & ATP-binding cassette sub-family B member 9 & 2.337 \\
P02671 & Fibrinogen $\alpha$ chain & 2.554 \\
P08311 & Cathepsin G & 2.763 \\
P02675 & Fibrinogen $\beta$ chain & 2.784 \\
\hline
\end{tabular}

S, paclitaxel-sensitive tissues; R, paclitaxel-resistant tissues.
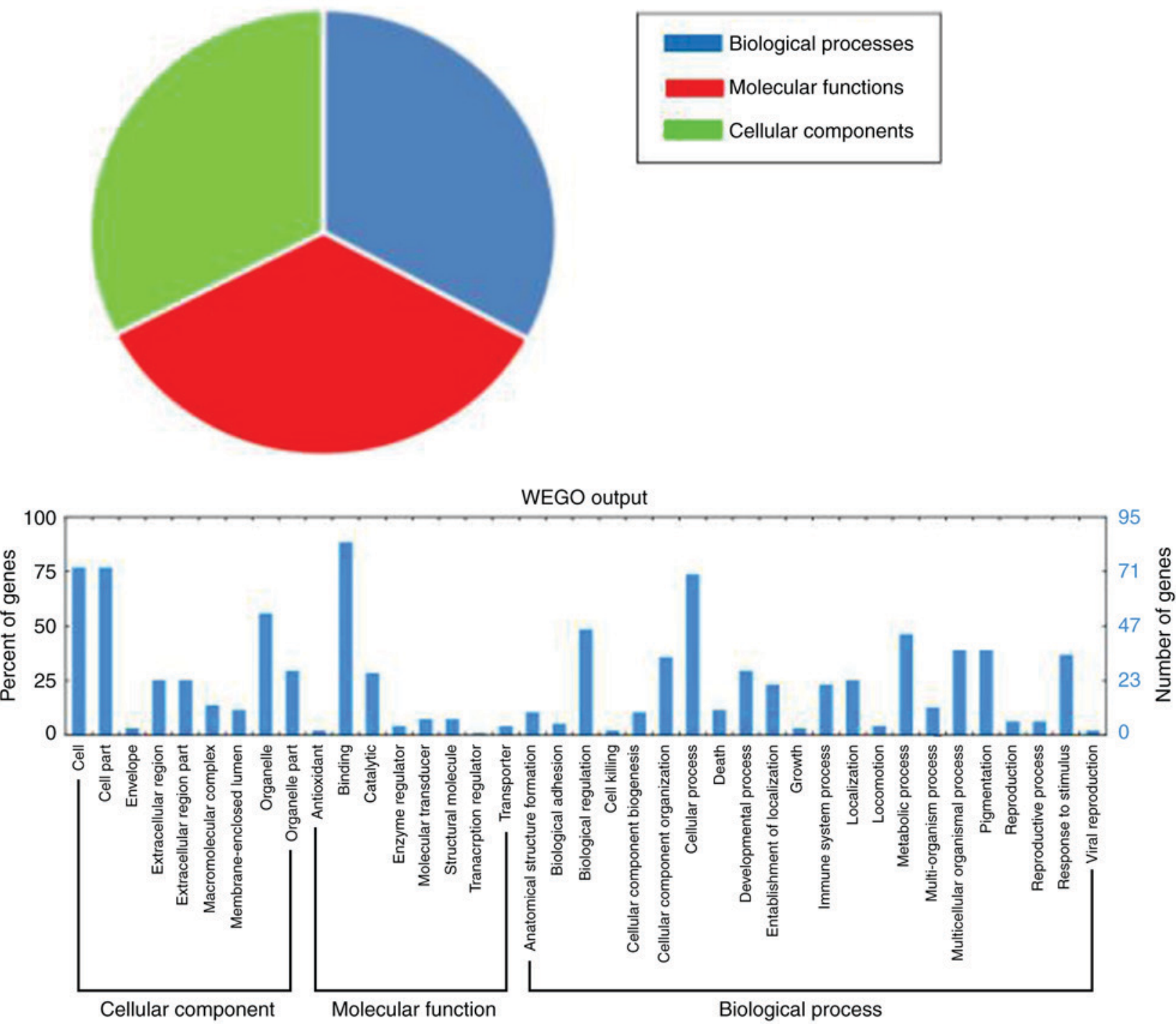

Figure 2. Gene Ontology enrichment analysis of the differentially expressed proteins.

Furthermore, the SIs of different tumor stages and differentiation grades were also significantly different (Fig. 1B and C).

iTRAQ assay. Proteins from paclitaxel-sensitive tissues and paclitaxel-resistant tissues were quantified by LC-MS/MS and iTRAQ analysis. In the present study, a total of 496 significantly differentially-expressed proteins were identified between paclitaxel-sensitive and paclitaxel-resistant tissues.
The threshold of the iTRAQ ratio (sensitive tissue/resistant tissue) was $<0.83$ or $>1.2$, which implied lower or higher expression of proteins in sensitive tissues compared with resistant tissues. Among them, 233 proteins were upregulated in the paclitaxel-resistant tissues and 263 proteins were downregulated. Certain proteins with important biological functions are listed in Table III. In order to investigate the functions of the differentially expressed proteins, Gene Ontology enrichment 


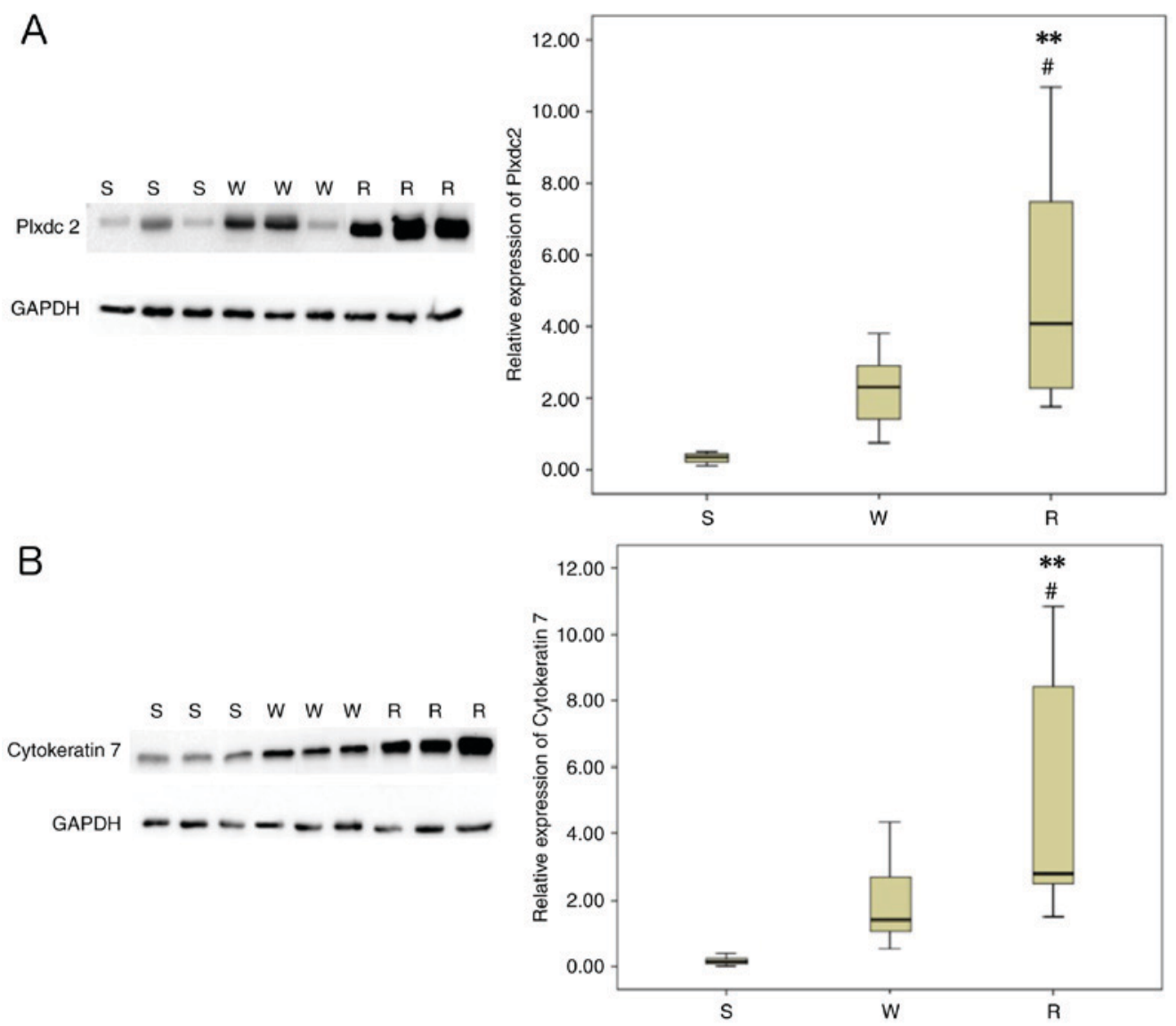

Figure 3. Verification of isobaric tags for relative and absolute quantitation by western blotting. (A) Plxdc2 protein expression in the paclitaxel-resistant tissues was significantly upregulated compared with the other types of tissue. (B) Protein expression of cytokeratin 7 in the paclitaxel-resistant tissues was significantly upregulated compared with the other types of tissue. ${ }^{* * *} \mathrm{P}<0.01 \mathrm{vs} . \mathrm{S},{ }^{\prime \prime} \mathrm{P}<0.05$ vs. W. Plxdc2, plexin domain containing 2 ; $\mathrm{S}$, sensitive; W, weak sensitive; R, resistant.

analysis was performed to analyze the functions of those proteins. A total of 96 differentially expressed proteins were divided into three categories: 'Molecular functions' (92.7\%), 'cellular components' (87.5\%), and 'biological processes' (88.5\%; Fig. 2).

Verification by western blot analysis. To validate the expression of the two selected proteins (Plxdc2 and CK7) identified by iTRAQ in EOC tissues with different chemosensitivities (sensitive, weakly sensitive and resistant), western blotting was performed and normalized densitometry data from the western blotting were used for the determination of relative expression values. Commercially available antibodies were used for probing the proteins, which were extracted from eight individuals with each type of tissues. The results were in concordance with those of the iTRAQ: the protein expression levels of Plxdc2 and CK7 were significantly increased in the paclitaxel-resistant tissues compared with the other two types of tissues (Fig. 3A and B).

\section{Discussion}

Ovarian cancer is the most lethal gynecologic malignancy in adult women (26). The standard treatment for EOC is surgical resection of the tumor mass, followed by a combination of paclitaxel and platinum. Although paclitaxel is effective as a first-line drug for advanced ovarian cancer, progression of the disease and mortality remain problems that originate from drug resistance. The main cause of paclitaxel resistance is thought to be the heterogeneity of the tumor tissue (27). EOC is biologically and morphologically heterogeneous, and it is possible to divide cases into several subtypes, which are then prescribed different treatments with different clinical outcomes (28). In the present study, an in vitro ATP-TCA, which has been widely used to determine the drug sensitivity of solid tumors, was used to assess heterogeneity in EOC. There was noticeable heterogeneity in chemosensitivity among the EOC samples examined: Highly- to mildly-differentiated or early-stage (I/II) EOC specimens had lower chemosensitivity to paclitaxel when compared with specimens with low differentiation or an advanced-stage (III), respectively. These results were consistent with those of a previous study, and implied that chemotherapy was not effective at preventing the recurrence of early-stage ovarian cancer (29).

In order to further screen the suitable biomarkers for predicting chemosensitivity to paclitaxel in ovarian cancer, the quantitative proteomic technique iTRAQ was performed to analyze the proteins from paclitaxel-resistant and paclitaxel-sensitive tissues. A total of 496 significantly differentially expressed proteins were identified, including 233 proteins which were upregulated and 263 proteins which 
were downregulated in paclitaxel-resistant tissues compared with paclitaxel-sensitive tissues. Two proteins of interest (Plxdc2 and CK7) were selected from among the upregulated proteins, which may be associated with paclitaxel resistance in EOC. The expression of Plxdc2 and CK7 in EOC tissues with different chemosensitivities (sensitive, weakly sensitive and resistant) was further detected by western blotting. The two proteins were revealed to be upregulated in the EOC tissues with paclitaxel resistance, consistent with the results from the iTRAQ analysis.

Plxdc2 has the ability to alter normal neurogenesis patterns, and is a novel mitogen for neural progenitors, and is present in the developing neural tube (30). Miller et al (31) were interested in Plxdc2 due to its protein architecture and expression pattern, and described the expression pattern of Plxdc2 in the developing mouse embryo. Notable similarities between the Plxdc2 expression multiple Wnt family members (Wnt1, Wnt3a, Wnt5a and Wnt8b) have been identified (32). In addition, Cheng et al (33) revealed that Plxdc2 is a cell-surface receptor for pigment epithelium derived factor (PEDF). PEDF is a secreted factor with multiple biological functions. It was initially considered to be a neurotrophic factor, but its recognized functions later expanded to include a stem cell niche factor, an inhibitor of cancer cell growth and, notably, the most potent natural antiangiogenic factor (34-36). A number of animal models have demonstrated the therapeutic value of PEDF in the treatment of blinding diseases and multiple types of cancer. Even in the presence of strong proangiogenic factors, PEDF is able to inhibit endothelial cell migration and angiogenesis. Furthermore, PEDF is a non-inhibitory member of the serine protease inhibitors (serpin) superfamily, which possesses potent physiological anti-angiogenic functions. PEDF decreases abnormal neovascularization by exerting anti-angiogenic effects which inhibit pro-angiogenic factors, including vascular endothelial growth factor, and this function has been investigated primarily in the eye and in cancer (37). In the present study, Plxdc2 expression was revealed to be upregulated in paclitaxel-resistant EOC tissues. Therefore, elucidating the associations between Plxdc2 and PEDF may lead to an improved understanding of the mechanisms and the development of novel therapeutic strategies for chemoresistant EOC.

CK7 is a simple, $\sim 55 \mathrm{kDa}$ epithelial cytokeratin which is primarily expressed in single-layered simple epithelia (38). Cytokeratins are intermediate cytoskeletal structural proteins present in the epithelial cells of the majority of organs, and are involved in mechanical support. They are also crucial for epithelial function, as cytokeratins are involved in signal transduction, cell polarity and gene regulation (39). They are maintained during carcinogenesis $(40,41)$. CK7 is expressed by a number of ductal and glandular epithelial cells (mainly gallbladder, hepatic ducts, and pancreatic ducts), by female genital tract tissues (ovary, endometrium, fallopian tube, and cervix) and by breast, lung, and urinary tract tissues (42). Chu et al (43) conducted immunohistochemistry to assess CK7 and cytokeratin 20 expression in 435 epithelial malignancy specimens, and 5\% stained cells was considered to be positive. Overall, $100 \%$ of lung, ovary, uterine and salivary gland cancers were CK7-positive. In addition, CK7 is a low molecular weight cytokeratin and its expression has been used to assess the differentiation of human primary and metastatic tumors of unknown origin $(44,45)$. In the present study, CK7 was revealed to be upregulated in paclitaxel-resistant EOC tissues, which may be involved in tumor metastasis and chemoresistance.

In conclusion, the mechanisms underlying paclitaxel resistance in ovarian cancer remain to be fully elucidated. Although further studies are required for large-scale validation of the candidate biomarkers identified by the present study, to the best of our knowledge the present study is the first to identify these candidate markers for paclitaxel-resistance in EOC. These results improve our understanding of the mechanisms underlying chemotherapy resistance and may help predict responses to targeted therapeutic agents. Furthermore, the identified proteins may aid further studies of the molecular mechanisms underlying paclitaxel treatment and resistance in EOC.

\section{Acknowledgements}

The present study was sponsored by the Capital Health Research and Development Projects of China (grant no. 2011-2008-05).

\section{References}

1. Cornelison R, Llaneza DC and Landen CN: Emerging Therapeutics to overcome chemoresistance in epithelial ovarian cancer: A mini-review. Int J Mol Sci 18, E2171, 2017.

2. Duda K, Cholewa H, Łabuzek K, Boratyn-Nowicka A and Okopień B: Novel strategies of ovarian cancer treatment. Pol Merku Lekarski 39: 337-342, 2015.

3. Ramalingam P: Morphologic, immunophenotypic, and molecular features of epithelial ovarian cancer. Oncology (Williston Park) 30: 166-176, 2016.

4. Bookman MA: Optimal primary therapy of ovarian cancer. Ann Oncol 27 (Suppl 1): i158-i162, 2016.

5. Matsumoto K, Onda T and Yaegashi N: Pharmacotherapy for recurrent ovarian cancer: Current status and future perspectives. Jpn J Clin Oncol 45: 408-410, 2015.

6. O'Donnell RL, Kaufmann A, Woodhouse L, McCormick A, Cross PA, Edmondson RJ and Curtin NJ: Advanced ovarian cancerdisplays functional intratumor heterogeneity that correlates to ex vivo drug sensitivity. Int J Gynecol Cancer 26: 1004-1011, 2016.

7. Tatar B, Boyraz G, Selçuk İ, Doğan AK, Usubütün A and Tuncer ZS: In vitro chemosensitivity in ovarian carcinoma: Comparison of three leading assays. J Turk Ger Gynecol Assoc 17: 35-40, 2016.

8. Zhang H, Zhang L, Wei L, Gao X, Tang LI, Gong W, Min NA, Zhang LI and Yuan Y: Knockdown of cathepsin L sensitizes ovarian cancer cells to chemotherapy. Oncol Lett 11: 4235-4239, 2016.

9. Lee M, Kim SW, Nam EJ, Cho H, Kim JH, Kim YT and Kim S: ATP-based chemotherapy response assay in primary or recurrent ovarian and peritoneal cancer. Yonsei Med J 55: 1664-1671, 2014.

10. Han SS, Choi SH, Lee YK, Kim JW, Park NH, Song YS, Lee HP and Kang SB: Predictive value of individualized tumor response testing by ATP based chemotherapy response assay in ovarian cancer. Cancer Invest 26: 426-430, 2008.

11. Zhao D, Zhang W, Li XG, Wang XB, Zhang LF, Li M, Li YF, Tian HM, Song PP, Liu J, et al: Predicting clinical chemo-sensitivity of primary ovarian cancer using adenosine triphosphate-tumor chemo-sensitivity assay combined with detection of drug resistance genes. Zhonghua Fu Chan $\mathrm{Ke} \mathrm{Za}$ Zhi 46: 193-198, 2011 (In Chinese).

12. Fehm T, Zwirner M, Wallwiener D, Seeger $H$ and Neubauer H: Antitumor activity of zoledronic acid in primary breast cancer cells determined by the ATP tumor chemosensitivity assay. BMC Cancer 12: 308, 2012. 
13. Neubauer H, Stefanova M, Solomayer E, Meisner C, Zwirner M, Wallwiener D and Fehm T: Predicting resistance to platinum-containing chemotherapy with the ATP tumor chemosensitivity assay in primary ovarian cancer. Anticancer Res 28: 949-955, 2008.

14. Safinya CR, Chung PJ, Song C, Li Y, Ewert KK and Choi MC: The effect of multivalent cations and Tau on paclitaxel-stabilized microtubule assembly, disassembly, and structure. Adv Colloid Interface Sci 232: 9-16, 2016

15. Tian Y, Tan AC, Sun X, Olson MT, Xie Z, Jinawath N, Chan DW, Shih IeM, Zhang $Z$ and Zhang H: Quantitative proteomic analysis of ovarian cancer cells identified mitochondrial proteins associated with paclitaxel resistance. Proteomics Clin Appl 3 : 1288-1295, 2009.

16. Gagné JP, Ethier C, Gagné P, Mercier G, Bonicalzi ME, Mes-Masson AM, Droit A, Winstall E, Isabelle M and Poirier GG: Comparative proteome analysis of human epithelial ovarian cancer. Proteome Sci 5: 16, 2007.

17. Tian Y, Yao Z, Roden RB and Zhang H: Identification of glycoproteins associated with different histological subtypes of ovarian tumors using quantitative glycoproteomics. Proteomics 11: 4677-4687, 2011.

18. Ling ZQ, Qi CJ, Lu XX, Qian LJ, Gu LH, Zheng ZG, Zhao Q, Wang S, Fang XH, Yang ZX, et al: Heterogeneity of chemosensitivity in esophageal cancer using ATP-tumor chemosensitivity assay. Acta Pharmacol Sin 33: 401-406, 2012

19. Konecny G, Crohns C, Pelgram M, Felber M, Lude S, Kurbacher C, Cree IA, Hepp H and Untch M: Correlation of drug response with the ATP tumorchemosensitivity assay in primary FIGO stage III ovarian cancer. Gynecol Oncol 77: 258-263, 2000

20. Wang H, Wu K, Liu Y, Wu Y and Wang X: Integrative proteomics to understand the transmission mechanism of Barley yellow dwarf virus-GPV by its insect vector Rhopalosiphum padi. Sci Rep 5: 10971, 2015.

21. Perkins DN, Pappin DJ, Creasy DM and Cottrell JS: Probability-based protein identification by searching sequence databases using mass spectrometry data. Electrophoresis 20: 3551-3567, 1999 .

22. Zhong XW, Zou Y, Liu SP, Yi QY, Hu CM, Wang C, Xia QY and Zhao P: Proteomic-based insight into Malpighian tubules of silkworm Bombyx mori. PLoS One 8: e75731, 2013

23. Shan N, Zhou W, Zhang S and Zhang Y: Identification of HSPA8 as a candidate biomarker for endometrial carcinoma by using iTRAQ-based proteomic analysis. Onco Targets Ther 9 : $2169-2179,2016$

24. Ashburner M, Ball CA, Blake JA, Botstein D, Butler H, Cherry JM, Davis AP, Dolinski K, Dwight SS, Eppig JT, et al: Gene ontology: Tool for the unification of biology. Nat Genet 25: 25-29, 2000

25. The Gene Ontology Consortium: Expansion of the Gene Ontology knowledgebase and resources. Nucleic Acids Res 45 D331-D338, 2017.

26. Christie M and Oehler MK: Molecular pathology of epithelial ovarian cancer. J Br Menopause Soc 12: 57-63, 2006.

27. Giordano S, Zucchetti M, Decio A, Cesca M, Fuso Nerini I, Maiezza M,Ferrari M, Licandro SA, Frapolli R, Giavazzi R, et al: Heterogeneity of paclitaxel distribution in different tumor models assessed by MALDI mass spectrometry imaging. Sci Rep 6: 39284, 2016.

28. Symeonides S and Gourley C: Ovarian cancer molecular stratification and tumor heterogeneity: A necessity and a challenge. Front Oncol 5: 229,2015.

29. Zhang $\mathrm{J}$ and Li H: Heterogeneity of tumor chemosensitivity in ovarian epithelial cancer revealed using theadenosine triphosphate-tumor chemosensitivity assay. Oncol Lett 9: 2374-2380, 2015.
30. Miller-Delaney SF, Lieberam I, Murphy P and Mitchell KJ: Plxdc2 is a mitogen for neural progenitors. PLoS One 6: e14565, 2011.

31. Miller SF, Summerhurst K, Rünker AE, Kerjan G, Friedel RH, Chédotal A, Murphy $\mathrm{P}$ and Mitchell KJ: Expression of Plxdc2/TEM7R in the developing nervous system of the mouse. Gene Expr Patterns 7: 635-644, 2007.

32. Qi W, Yang C, Dai Z, Che D, Feng J, Mao Y, Cheng R, Wang Z, He X, Zhou T, et al: High levels of pigment epithelium-derived factor in diabetes impair wound healing through suppression of Wnt signaling. Diabetes 64: 1407-1419, 2015.

33. Cheng G, Zhong M, Kawaguchi R, Kassai M, Al-Ubaidi M, Deng J, Ter-Stepanian M and Sun H: Identification of PLXDC1 and PLXDC2 as the transmembrane receptors for the multifunctional factor PEDF. Elife 3: e05401, 2014.

34. Sagheer U, Gong J and Chung C: Pigment Epithelium-Derived Factor (PEDF) is a determinant of stem cell fate: Lessons from an Ultra-Rare disease. J Dev Biol 3: 112-128, 2015.

35. Zhang S, Zhai G, Shi W, Wang Y, Zhu L, Dai Y and Chen C: Pigment Epithelium-Derived factor inhibits oxygen-induced retinal neovascularization in a murine model. Fetal Pediatr Pathol 35: 173-185, 2016.

36. Belkacemi L and Zhang SX: Anti-tumor effects of Pigment Epithelium-Derived Factor (PEDF): Implication for cancer therapy. A mini-review. J Exp Clin Cancer Res 35: 4, 2016.

37. Chuderland D, Ben-Ami I, Bar-Joseph H and Shalgi R: Role of pigment epithelium- derived factor in the reproductive system. Reproduction 148: R53-R61, 2014

38. Sandilands A, Smith FJ, Lunny DP, Campbell LE, Davidson KM, MacCallum SF, Corden LD, Christie L, Fleming S, Lane EB and McLean WH: Generation and characterisation of Keratin 7 (K7) knockout mice. PLoS One: e64404, 2013.

39. Windoffer R, Beil M, Magin TM and Leube RE: Cytoskeleton in motion: The dynamics of keratin intermediate filaments in epithelia. J Cell Biol 194: 669-678, 2011.

40. Bayrak R, Yenidunya S and Haltes H: Cytokeratin 7 and Cytokeratin 20 expression in colorectal adenocarcinoma. Pathol Res Pract 207: 156-160, 2011

41. Gurzu S and Jung I: Aberrant pattern of the cytokeratin 7/cytokeratin 20 immunophenotype in colorectal adenocarcinomas with BRAF mutation. Pathol Res Pract 208: 163-166, 2012.

42. Toyoshima M, Momono Y, Makino H, Kudo T, Oka N, Sakurada J, Suzuki H, Kodama H and Yoshinaga K: Cytokeratin 7-positive/cytokeratin 20-negative cecal adenocarcinoma metastaticto the uterine cervix: A case report. World J Surg Oncol 14: 22, 2016.

43. Chu P, Wu E and Weiss LM: Cytokeratin 7 and cytokeratin 20 expression in epithelial neoplasms: A survey of 435 cases. Mod Pathol 13: 962-972, 2000

44. Shin JH, Bae JH, Lee A, Jung CK, Yim HW, Park JS and Lee KY: CK7, CK20, CDX2 and MUC2 Immunohisto-chemical staining used to distinguish metastatic colorectal carcinoma involving ovary from primary ovarian mucinous adenocarcinoma. Jpn J Clin Oncol 40: 208-213, 2010.

45. Moll R, Divo M and Langbein L: The human keratins: Biology and pathology. Histochem Cell Biol 129: 705-733, 2008.

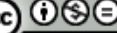

This work is licensed under a Creative Commons Attribution-NonCommercial-NoDerivatives 4.0 International (CC BY-NC-ND 4.0) License. 